\title{
Nav1.6 in the vasomotor center is a promising key molecule in the pathophysiology of stress-induced hypertension
}

\author{
Takuya Kishi ${ }^{1}$
}

Received: 12 October 2018 / Revised: 19 October 2018 / Accepted: 19 October 2018 / Published online: 4 December 2018

(c) The Japanese Society of Hypertension 2018

Stress is undoubtedly a risk factor for the development of various diseases, including cardiovascular diseases, mental disorders, malignant tumors, diabetes mellitus, obesity, metabolic syndrome, and inflammatory, and autoimmune disorders [1]. Psychosocial stressors are known to be the most powerful and to exert profound effects on cardiovascular diseases. Moreover, it has already been established that sympathoexcitation in the hypothalamic-pituitaryadrenocortical axis is a main characteristic of the stress response, which is an adaptation to maintain homeostasis [1]. When stressors are too frequent and/or too intensive, this adaptation can in turn lead to various diseases, including hypertension [1]. However, there is still conflicting information about the causal relation between stress and hypertension. The results of experimental studies focusing on the mechanisms of stress-induced hypertension include both positive and negative evidence of the participation of stress in the development of hypertension [1]. Moreover, although previous studies have clearly indicated that sympathetic nerve activity is determined by the rostral ventrolateral medulla (RVLM) as the vasomotor center [2], molecular abnormalities in the RVLM in relation to stressinduced hypertension remain unclear.

In this context, Jia-Xiang $\mathrm{Wu}$ et al. have published a suggestive study, "Upregulation of Nav1.6 expression in the rostral ventrolateral medulla of stress-induced hypertensive rats," in Hypertens Res [3]. They focused on the voltage-gated sodium channel Nav1.6, which has been established to contribute to neuronal hyperexcitability, and hypothesized that the expression of Nav1.6 would be upregulated in the RVLM with sympathoexcitation in a stress-induced hypertensive model

Takuya Kishi

tkishi@cardiol.med.kyushu-u.ac.jp

1 Department of Advanced Risk Stratification for Cardiovascular Diseases, Center for Disruptive Cardiovascular Medicine, Kyushu University, Fukuoka, Japan induced by administering electric foot-shocks and noises. In the experiment, three important results were shown: (1) Nav1.6 protein expression in the RVLM, systolic blood pressure, and renal sympathetic nerve activity in stress-induced hypertensive rats were higher than those of control rats, peaking at the tenth day of stress; (2) upregulation of Nav1.6 expression in neurons was strongly correlated with systolic blood pressure; and (3) knockdown of Nav1.6 in the RVLM of the stress-induced hypertensive rats decreased systolic blood pressure, heart rate, and renal sympathetic nerve activity. These results strongly indicated that upregulation of Nav1.6 expression in RVLM neurons in stress-induced hypertensive rats contributes to overactivation of the sympathetic system in the development of stress-induced hypertension. The presently described study is the first experiment to assess the pathophysiological function of Nav1.6 in the RVLM of stressinduced hypertension. Several novel clinical perspectives have been provided to us. First, sympathoexcitation mediated by neuronal activity in the RVLM is the major inducing factor of stress-induced hypertension. We should focus on the pathophysiological role of the sympathetic nervous system in hypertension. Second, the voltage-gated sodium channel Nav1.6 in the RVLM is closely related to systolic blood pressure with sympathoexcitation in stress-induced hypertension. Because molecular abnormalities in the vasomotor center in stress-induced hypertension have not been fully clarified, the described results contribute to the future progression of research on hypertension. Finally, regarding the clinical implications, central Nav1.6 could be a novel potential clinical marker or therapeutic target for stress-induced hypertension.

The RVLM is known as the vasomotor center that determines the basal central sympathetic outflow [2]. There are inputs into the RVLM from baroreceptors, chemoreceptors, and visceral receptors via the nucleus of the solitary tract and the paraventricular nucleus of the hypothalamus (PVN). In this decade, we have demonstrated that angiotensin II-induced oxidative stress and/or inflammation in the RVLM causes sympathoexcitation in stroke-prone spontaneously hypertensive rats (SHRSP) [2]. In contrast, 
nitric oxide decreases sympathetic nerve activity via the release of gamma-amino butyric acid, an inhibitory amino acid, at presynaptic neurons in the RVLM of SHRSP [2]. Regarding RVLM and stress-induced hypertension, a recent report demonstrated that upregulation of angiotensin II in the RVLM causes stress-induced hypertension by increasing excitatory and suppressing inhibitory amino acid release [4]. Another report indicated that Nav1.6 induces the release of excitatory amino acids at presynaptic neurons [5]. The presently described study is compatible with these previous studies, and Nav1.6 could be a key molecule regulating excitatory and inhibitory amino acids in the RVLM in stress-induced hypertension.

In the pathophysiology of stress-induced hypertension, baroreflex failure should be involved [1]. The baroreflex is the fastest negative feedback system to stabilize blood pressure. Arterial baroreceptors are stretch receptors located within the arterial wall of elastic vessels, such as the aortic arch and carotid sinuses. The baroreflex senses blood pressure, and activated afferent nerves relay the pressure signal to the vasomotor center. The vasomotor center changes the mechanical properties of the ventricle and vascular system to stabilize blood pressure by modulating the autonomic nervous system [6]. Short-term blood pressure variability is worsened by baroreflex failure [6], and sympathoexcitation responses to mental stress blunt baroreflex function in posttraumatic stress disorder [7]. Previously, we demonstrated that decreased inhibitory amino acids and increased excitatory amino acids in the RVLM worsen baroreflex function [2]. Considering these previous studies, the presently described study provides further deep insights into the molecular and physiological mechanisms in the RVLM in stress-induced hypertension.

Interactions between neurons and glial cells have been shown to be involved in various diseases. Regarding stressinduced hypertension, a recent study has suggested that neuroinflammation with microglial activation in the RVLM plays a role in the development of stress-induced hypertension by increasing excitatory and suppressing inhibitory amino acid release [8]. We also demonstrated that microglial activation in the PVN was associated with the development phase, not the established phase, of hypertension [9]. Furthermore, selective deletion of the angiotensin receptor at astrocytes decreases sympathetic nerve activity in ischemiainduced heart failure [9]. Interestingly, Nav1.6 expression is increased in reactive astrocytes in an epilepsy model associated with neuronal hyperexcitability [10]. Combined, the results of these previous studies and the presently described experiment show that Nav1.6 in the RVLM is a potential key molecule in the pathophysiology of disrupted sympathetic regulation mediated by neuron and glial interactions.

In conclusion, Jia-Xiang $\mathrm{Wu}$ et al. showed novel molecular mechanisms in the RVLM in stress-induced hypertension. Nav1.6 in neurons of the RVLM could be a promising therapeutic target for stress-induced hypertension. Future and further experiments on the mechanisms of the stress-induced upregulation of Nav1.6 in the RVLM and on the time dependence of Nav1.6 expression in the RVLM in stress-induced hypertension are expected.

\section{Compliance with ethical standards}

Conflict of interest The author declares no conflict of interest

\section{References}

1. Puzserova A, Bernatova I. Blood pressure regulation in stress: focus on nitric oxide-dependent mechanisms. Physiol Res. 2016;65:S309-42.

2. Kishi T. Regulation of sympathetic nervous system by nitric oxide and oxidative stress in the rostral ventrolateral medulla: 2012 Academic Conference Award from the Japanese Society of Hypertension. Hypertens Res. 2013;36:845-51.

3. Wu JX, Tong L, Hu L, Xia CM, Li M, Chen QH, et al. Upregulation of Nav1.6 expression in the rostral ventrolateral medulla of stress-induced hypertensive rats. Hypertens Res. 2018;41:1013-22.

4. Du D, Chen J, Liu M, Zhu M, Jing H, Fang J, et al. The effects of angiotensin II and angiotensin-(1-7) in the rostral ventrolateral medulla of rats on stress-induced hypertension. PLoS ONE 2013;8:e70976.

5. Mi Y, Wu Q, Yuan W, Chen F, Du D. Role of microglia M1/M2 polarisation in the paraventricular nucleus: new insight into the development of stress-induced hypertension in rats. Auton Neurosci. 2018;213:71-80.

6. Kishi T. Baroreflex failure and beat-to-beat blood pressure variation. Hypertens Res. 2018;41:547-52.

7. Park J, Marvar PJ, Liao P, Kankam ML, Norrholm SD, Downey RM, et al. Baroreflex dysfunction and augmented sympathetic nerve responses during mental stress in veterans with posttraumatic stress disorder. J Physiol. 2017;595:4893-908.

8. Du D, Hu L, Wu J, Wu Q, Cheng W, Guo Y, et al. Neuroinflammation contributes to autophagy flux blockade in the rostral ventrilateral medulla in stress-induced hypertension rats. J Neuroinflamm. 2017;14:169.

9. Kishi T. Heart failure as a disruption of dynamic circulatory homeostasis mediated by brain. Int Heart J. 2016;57:145-9.

10. Zhu $\mathrm{H}$, Zhao $\mathrm{Y}, \mathrm{Wu} \mathrm{H}$, Jiang $\mathrm{N}$, Wang $\mathrm{Z}$, Lin $\mathrm{W}$, et al. Remarkable alterations of Nav1.6 in reactive astrogliosis during epileptogenesis. Sci Rep. 2016;6:38108. 